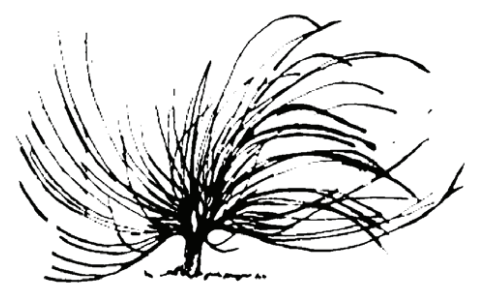

\title{
La Pizarra Digital Interactiva: Componentes, Configuraciones, Posibilidades y Singularidades
}

\author{
Rolando Aguilar Álvarez ${ }^{1}$ \\ Universidad Nacional \\ Heredia, Costa Rica \\ rolando.aguilar.alvarez@una.cr \\ Alberto Ramírez Martinell ${ }^{2}$ \\ Universidad Veracruzana \\ México \\ albramirez@uv.mx
}

\begin{abstract}
Resumen
En los años recientes, las Pizarras Digitales Interactivas (PDI) han ido ganando un espacio en las aulas de los docentes universitarios. En este trabajo hacemos una revisión de las PDI a partir de sus componentes esenciales, sus implicaciones en el proceso de enseñanza-aprendizaje en las Instituciones de Educación Superior (IES) y algunas de sus posibles configuraciones en el aula. Asimismo, reflexionamos en torno al análisis de las posibilidades que brinda la existencia de dichos componentes en las aulas de las IES. De esta manera -y analizando
\end{abstract}

\section{(c) $(1) \Theta$}

Recibido: 4 de noviembre de 2014-Aprobado: 13 de abril de 2015

1 Máster en Administración de Proyectos por la UCI, Costa Rica. Lic. en Computación e Informática por la UCR, Costa Rica. Actual Académico del Programa UNA Virtual, Universidad Nacional de Costa Rica

2 Doctor en Tecnología Educativa por la Universidad de Lancaster, Inglaterra, miembro del sistema mexicano de investigadores (SNI) y actual coordinador de la maestría en educación virtual. 
situaciones concretas de uso- comparamos las singularidades de diferentes configuraciones de componentes y sus implicaciones para una reflexión informada sobre su integración en las IES como recursos didácticos útiles y justificados.

Palabras clave: pizarras digitales interactivas, educación superior, integración de las TIC

\begin{abstract}
In recent years, Interactive Whiteboards (IWB) have gained a respected space within the academic practitioners in the university context. In this article, we will do a revision of these devices going from their main components, their implications in the teaching-learning process within Higher Education Institutions (HEI) and the way they can be configured in the classroom. We also reflect on the academic possibilities generated from their use and how their components may enhance the classroom experience. In this way -and reflecting upon given specific situations- we compare the singularities of different configurations of the IWB components and the implications for their integration in HEI as justified and useful didactic resources.
\end{abstract}

Keywords: digital interactive whiteboards, higher education, ICT integration

\title{
Introducción
}

Las Tecnologías de Información y Comunicación (TIC) han llegado a cada uno de los distintos escenarios de la vida moderna y las aulas de instituciones de educación superior (IES) no son la excepción. Las vías de entrada de las TIC a las IES han sido diversas, por ejemplo, a través de las plataformas de educación a distancia, los programas multimedia de apoyo a la enseñanza, los sistemas de evaluación de respuestas en el aula, el software y bases de datos especializados, el uso de los dispositivos móviles como soporte educativo y las pizarras digitales interactivas, entre otros. En el espacio universitario, podemos destacar el desarrollo de modelos educativos semipresenciales y virtuales que han 
sido usados como complemento - o sustituto- de la modalidad tradicional (ver Ramírez-Martinell, Maldonado, 2015); además, de la adopción de artefactos -de hardware y de software- cuyos objetivos primordiales son simplificar la labor docente, ampliar los canales de comunicación y proveer a los estudiantes y profesores de herramientas adecuadas para su desempeño académico.

Es difícil enumerar esta serie de artefactos ya que además de cada día surgen nuevas tecnologías que dotan a los docentes de vías innovadoras para mejorar las experiencias de aprendizaje de sus estudiantes, la cultura disciplinar va determinando tanto a los usos como a los mismos artefactos tecnológicos. Por distintos motivos, el medio universitario ha sido campo fértil para la adopción y popularización de Internet y otras soluciones digitales, que han favorecido la exploración de diversas potencialidades de estos medios como recursos educativos. De hecho, las universidades más reconocidas en el mundo han dedicado departamentos y esfuerzos institucionales significativos a la elaboración de recursos digitales, cursos en línea e inclusive al desarrollo de plataformas y repositorios que permiten la socialización del contenido que desarrollan. Las universidades modernas actualmente cuentan con una activa presencia en Internet tanto para presentarse en línea y ofrecer sus servicios educativos, como para utilizar Internet y las plataformas distribuidas como medios educativos para la atención a un mayor número de estudiantes.

Cabe mencionar que las TIC e Internet no solamente han impactado la labor docente sino también otras actividades universitarias como la investigación, la extensión universitaria y la relación con las empresas, además del mantenimiento de contacto con los egresados, la vinculación de docentes y estudiantes con otras IES y la colaboración en la ejecución de experimentos remotos, compartiendo recursos locales del campus o de otras entidades geográficamente inalcanzable pero digitalmente a la mano. Las ventajas del uso de las TIC e Internet en el aula son diversas y difíciles de enumerar, pero para un mejor uso en un contexto áulico, la ejecución de programas para mostrar material multimedia, simular procesos complicados de traer o replicar en el aula hacer presentaciones electrónicas o buscar información requieren de un dispositivo de visualización distinto al monitor convencional de la computadora, cuyo tamaño no sólo no permite un uso cómodo de la tecnología para un grupo grande de estudiantes sino que podría resultar 
contraproducente y la posibilidad de tener una computadora para cada estudiante encarece y complica el equipamiento del aula. Ante esta situación, pareciera inminente contar con un monitor prácticamente del tamaño de una pizarra tradicional (PT) para poder hacer uso eficiente de las TIC en el aula.

La PT es sin lugar a dudas, uno de los medios de instrucción más importantes que tiene a su disposición el docente y consideramos que es interesante reflexionar sobre qué es lo que pasa cuando se dota de tecnología digital a una PT. Por eso, en este texto nos concentramos en el análisis de las posibilidades que pueden brindar las PT enriquecidas con TIC como soporte al proceso educativo, porque creemos que sus potencialidades podrían no estar completamente comprendidas y valoradas; de ser así, por un lado, se podrían obviar algunas consideraciones propicias de uso; pero por el otro, también corremos el riesgo de sobrevalorar al dispositivo y sus usos redundando en un exceso de entusiasmo que podría preceder a una decepción sobre las posibilidades de las Pizarras Digitales Interactivas (PDI). Consideramos que un análisis imparcial de las posibilidades de las PDI además de ser un aporte importante para el empleo justificado de este tipo de dispositivo, servirá para la discusión y reflexión sobre el uso de las PDI, su necesidad y justificación en las IES y en un momento dado para planificar y mesurar las inversiones institucionales de su adquisición masiva. Finalmente, vale la pena mencionar que este trabajo también aporta a la discusión y análisis de cómo las TIC pueden lograr la mejora de la visualización y la interacción con los contenidos a través de nuevas formas de presentación (Passey, 2009).

\section{La pizarra digital interactiva: lo que dice la literatura}

Aunque no existe una definición estándar sobre qué es una Pizarra Digital Interactiva (PDI), autores como Marques (2006), Noda (2009) y Gallego, Cacheiro y Dulac (2009), la definen a partir de sus componentes, haciendo énfasis en las diferencias entre las PDI y las Pizarras Digitales (PD). Para Gallego, Cacheiro y Dulac (2009) la PD es un sistema tecnológico, generalmente integrado por una computadora $\mathrm{y}$ un video-proyector, que permite proyectar contenidos digitales en un formato idóneo para la visualización en un salón de clase, favoreciendo la interacción del grupo con las imágenes proyectadas y utilizando los 
periféricos de la computadora (mouse, teclado, etc.). Mientras que la PDI - para ellos mismos- es un sistema tecnológico, generalmente integrado por una computadora, un video-proyector (o cañón de proyección) y un dispositivo de control de puntero, que permiten proyectar "en una superficie interactiva" contenidos digitales en un formato idóneo para la presentación grupal, con quienes además se podrá interactuar directamente sobre la superficie de proyección.

Marques (2006) dice que una PD se convierte en una PDI cuando se agrega un mural táctil en el que por un lado se pueden hacer anotaciones y por otro se puede controlar la computadora mediante el marcador de pizarrón especial de la PDI, generando al mismo tiempo una experiencia hasta cierto punto inmersiva. De manera similar, Noda (2009) comenta que la interacción físico-digital se logra a través de la superficie que se proyecta y aclara que puede hacerse por medio de una pantalla táctil, un puntero o marcador que permita escribir y marcar en la superficie o inclusive con los dedos. En ocasiones, esta precisión entre las posibles configuraciones de la PDI puede ser significativa. Por ejemplo, Gallego, Cacheiro y Dulac (2009) han observado que las interfaces táctiles son más simples para niños pequeños o de educación especial debido a que la interacción con sistemas digitales a través de cualquiera de estos medios es más cómoda y natural y que el uso del mouse o del teclado tenderán a ser relegados (Marques, 2006). La diferencia esencial entre PD y PDI radica entonces en la existencia de un mecanismo que permite la interacción sobre la superficie de proyección prescindiendo del uso directo de una computadora y sus dispositivos de entrada.

Gallego, Cacheiro y Dulac (2009) aclaran que hay autores que no diferencian entre PD y PDI, en consecuencia, esta omisión podría impactar en los costos de inversión y en las posibilidades de uso directo en el aula. La diferencia fundamental de costos estaría asociada al dispositivo que permite controlar la computadora actuando directamente sobre la superficie proyectada, pues este es el componente que marca la diferencia y la diferencia en su uso podría tener implicaciones logísticas y también didácticas.

Las PDI han recibido notable atención en los últimos años. Marques y Casals (2002) las consideraron como una de las tres bases tecnológicas de la escuela del futuro. El mismo Marques (2006) consideró que de los recursos tecnológicos que los docentes tienen a su alcance la "pizarra digital constituye, sin duda, la que proporciona un mayor 
potencial didáctico, al tiempo que induce una progresiva introducción de prácticas innovadoras y centradas en la actividad del estudiante" para un contexto áulico, inclusive la PDI puede ser considerada como uno de los mejores instrumentos de apoyo para la renovación pedagógica en las aulas tradicionales. La PDI trae al salón de clases, una serie de recursos tecnológicos poderosos en un formato que resulta familiar para profesores y estudiantes: la clase total con exposición en la pizarra. En la literatura podemos encontrar que a las PDI también se les denomina Pizarras Interactivas, digital whiteboard; interactive whiteboard (IWB) (Domingo y Marques, 2011) o pizarra electrónica (Marques, 2006).

Las PDI tienen diversas potencialidades para incidir de manera positiva en el proceso de enseñanza-aprendizaje (Glover, Miller, Averis y Door, 2007), de las cuales podemos comentar que, su semejanza con los sistemas tradicionales de enseñanza permiten que las TIC entren al aula de un modo suave y poco intrusivo (Gallego, Cacheiro y Dulac, 2009); asimismo promueven un comportamiento activo de los estudiantes (Hervás, Toledo y González, 2010). Sin embargo, Armstrong, Barnes, Sutherland, Curran, Mills y Thompson (2005) consideran que existen incertidumbres sobre la concreción de estas posibilidades, pues consideran que las investigaciones sobre las PDI aún están en sus etapas iniciales y que vistas desde un enfoque propio de la teoría sociocultural en el que las acciones humanas deben ser concebidas como actos mediados por herramientas -incluyendo a las computadoras, los bolígrafos, los punteros, el papel, los diagramas y hasta el lenguaje-, resulta evidente que el estudio de las relaciones entre profesores, estudiantes, objetos tecnológicos y lo que se puede hacer con ellos es un área de oportunidad para conducir investigaciones al respecto; y en ese mismo tren de pensamiento, Glover, Miller, Averis y Door (2005) reconocen que hay poca investigación sobre el impacto real de estas tecnologías en el contexto académico y atribuir cambios en la enseñanza o el aprendizaje es aún prematuro. Aún cuando algunos autores reconocen que las PDI pueden tener potencialidades mayores que las pizarras tradicionales, el desempeño de profesores y estudiantes no necesariamente se modifica o mejora con tan solo utilizarlas (Armstrong, Barnes, Sutherland, Curran, Mills, Thompson, 2005); al respecto, Glover, Miller, Averis y Door (2007) mencionan que el uso de esta tecnología por sí sola no conduce a un mejor aprendizaje y Quiroz (2012) plantea que disponer de una PDI es como disponer de un instrumento que para ser empleado 
de manera adecuada se deberá saber sus implicaciones y formas de uso. Passey (2009), al realizar un análisis de situaciones en las que la incertidumbre del impacto de las PDI, precisa que no siempre se puede saber a ciencia cierta lo que realmente aporta una tecnología o medio a los procesos de enseñanza o de aprendizaje.

La adopción de PDI, al igual que la de otras tecnologías, se puede basar ya sea en una motivación de modernización áulica o en la integración de tecnología digital con fines educativos concretos en los que el contenido y el objetivo de la clase sean los que permitan a los profesores, instituciones y hacedores de políticas tomar las decisiones adecuadas más allá de las modas y tendencias tecnológicas. Quiroz (2012) dice que es importante mantener el foco en el objetivo de la clase y no en la tecnología en sí, se debe anticipar que sucederá cuando la novedad pase y la tecnología se quede en el aula (Glover, Miller, Averis y Door, 2007). Por eso, hay que ver a la tecnología digital como un elemento adicional en el aula que deberá optimizar procesos ya sean de comunicación, de gestión o de acceso a la información y su integración en este espacio académico deberá hacerse de manera reflexiva y justificada y no a partir de un discurso político y de modernización de la infraestructura institucional. No obstante, no debe ignorarse que hay situaciones que pueden invalidar el uso potencial de las PDI, como pueden ser la necesidad de recursos financieros para la compra de los equipos, la necesidad de contar con la infraestructura energética y de conectividad, la necesidad de capacitar a los docentes, la potencial aparición de problemas técnicos y de soporte, entre otros.

Como cualquier tecnología, las características intrínsecas de las PDI pueden derivar también en desventajas si no se resuelven situaciones previas a la adopción, como la alta dependencia de energía eléctrica pues sin ella la PDI no funciona, lo cual limita su empleo respecto a las pizarras tradicionales que no necesitan energía. Adicionalmente, en el edificio donde se instalen las PDI debe existir una infraestructura adecuada para colocarlas, en ocasiones, se deberá considerar una conexión a Internet, la iluminación para que su reflejo no afecte la legibilidad de lo que se escriba en la PDI, la disposición del aula podrá predeterminar la configuración permanente del acomodo de las sillas, por ejemplo, si hubieran varias PDI, el salón tendría que disponerse de modo que facilite el trabajo en equipos y la interacción con el dispositivo. Quizás las instituciones que cuenten con pequeñas PT portátiles que pueden 
moverse por el salón de clases, dispongan de un mayor grado de flexibilidad para la configuración del salón. Otra de las limitaciones al uso de las PDI, es la necesidad institucional de entrenar a los docentes, que tendrían que habituarse a la nueva forma de interactuar, aunque guarda semejanzas con las PT no es exactamente igual. En este mismo sentido, se agrega la posibilidad de aparición de más problemas técnicos potenciales, derivados de la mayor sofisticación de las PDI respecto a las PT. En cierta medida, el docente deberá utilizar el dispositivo controlador y el software que lo acompaña para el mejor manejo de la pizarra, lo cual podría implicar una resistencia al autoaprendizaje o a la capacitación continua institucionalizada.

Quizás la limitación más importante de las PDI, es el acceso al equipo, que está ligado con un costo inicial y en algunos casos con la actualización del software que acompaña al dispositivo apuntador. Esta inversión puede ser una limitación importante para la adopción de las PDI, no solo por los costos sino por la dependencia que se genera con el software y con el proveedor de tecnología. Aún cuando las limitaciones se pueden superar, no es correcto obviarlas. Será la disposición tecnológica institucional y personal las que permitan aliviar estas y otras limitaciones.

\section{PDI: Configuraciones y Características}

Como pudo entenderse en la sección anterior, las PDI pueden integrarse al espacio académico de diferentes formas y disponiendo de todos o de algunos de sus componentes. Las PDI están compuestas por distintos elementos que pueden o no estar presentes en distintos arreglos o configuraciones, por ejemplo, dotar al profesor de una computadora y sus accesorios, de un dispositivo de proyección y de una superficie de proyección interactiva para impartir su clase.

No hay un consenso en los autores sobre qué elementos deben estar presentes de manera obligatoria para que una configuración tecnológica se considere como PDI; o si son extensiones al concepto básico. A manera de ejemplo, Domingo y Marques (2011) le llaman Aula 2.0 al espacio escolar dotado de una PDI y de computadoras con acceso a Internet. Las PDI tienen principalmente tres características que involucran la proyección, el contenido y la conectividad con Internet: 
1. la superficie de proyección debe ser de "gran tamaño" (Marques,2006);

2. deben fomentar el uso de contenido multimedia según Marques (2006);

3. deben estar conectados a Internet (Marques, 2006; Noda, 2009; Domingo y Marques, 2011).

No obstante, las PDI pueden considerar otros periféricos como el apuntador interactivo -periférico de entrada que permite controlar la computadora desde la superficie proyectada-. Esta posibilidad es importante analizarla en detalle, pues no solo pasa por la existencia -o no- de los componentes sino por las sinergias positivas o negativas que se producen entre ellos.

La introducción en el aula de una configuración tecnológica como la PD y la PDI no implica ninguna alteración a la esencia del modelo de enseñanza de aprendizaje tradicional, sino una mejora. Con la Pizarra Tradicional (PT), el docente era capaz de presentar ideas, situaciones y conceptos apoyándose de una superficie en la que podía escribir y hacer diagramas. Con la PD y la PDI, la dinámica de grupo se mantiene pero trayendo al aula un contenido interactivo que usualmente solo se puede ver en el monitor de una computadora. La limitación en el tamaño del monitor es una de las causas que justifica el uso de las PD; ya que permiten desplegar en una superficie lo suficientemente amplia y visible para el grupo, todo lo que se hace en la computadora o dispositivo móvil. Sin embargo, la PD trae consigo un reto al uso de las PT: si se usa la superficie de la PT para proyectar contenido, el uso compartido puede ser complejo pues habría que ocultar la proyección cuando se requiera escribir al estilo tradicional y limpiar la PT cuando se requiera seguir proyectando.

Cuando las aulas disponen de espacio para tener una superficie de proyección de la PD que no se solape con la PT, se puede contar con la posibilidad de emplear toda la capacidad de la computadora al vista de todo el grupo pero conservando el estilo de una PT. En este contexto $(\mathrm{PD}+\mathrm{PT})$, se pueden establecer diversas estrategias pedagógicas similares a las PDI. Sin embargo, es importante marcar algunas diferencias de ese contexto $\mathrm{PT}+\mathrm{PD}$ respecto a una PDI: 
- $\quad$ La forma en que se escribe libremente en una PT (tiza, marcador) y la forma de interactuar con la PD (mouse y teclado) es diferente y habría que estar cambiando entre ellos constantemente. La PDI resuelve este problema porque unifica ambas formas de interacción.

- Lo que se escribe en la PT no puede almacenarse mientras que lo que se escribe en la PDI sí es posible hacerlo.

- $\quad$ En la PDI se tiene una sola área de trabajo, lo cual permite concentrar en ella la atención de los estudiantes, reducir la necesidad de movimiento del profesor y no exigir un espacio tan grande como lo obligaría la combinación PD + PT.

Adicionalmente a las configuraciones anteriores, en ocasiones, se puede disponer de recursos analógicos o digitales diversos que podrían ser combinados dependiendo del objetivo de la clase. Para Gallego, Cacheiro y Dulac (2009), la configuración de PDI adecuada puede aumentar las posibilidades para el trabajo grupal y para la comunicación intergrupal propiciando nuevas formas de interacción entre los actores universitarios.

Son diversas las posibles configuraciones que se pueden establecer a partir de la presencia -o no- de los distintos componentes de que disponen los profesores (pizarra tradicional, video-proyector, computadora, bocinas, PDI, acceso - o no- a Internet, cámara de video, etc.); y aun cuando en este texto hagamos una propuesta arbitraria al proponer un número finito de configuraciones, la razón nos justifica, ya que a partir de esta propuesta haremos un análisis de viabilidad de uso de las PDI en el aula. Las seis configuraciones que proponemos inicialmente son:

- $\quad$ Configuración Pizarra Tradicional (PT): Pizarra tradicional con sus accesorios (borrador y marcador o rotulador)

- Configuración Pizarra Digital (PD): PD (computadora, video-proyector) y acceso a Internet.

- $\quad$ Configuración Pizarra Digital + Pizarra Tradicional (PD+PT): PD (computadora, video-proyector) y acceso a Internet, además de una pizarra tradicional con sus accesorios.

- $\quad$ Configuración Laboratorio (L): Laboratorio con computadoras con acceso a Internet, sin pizarra de ningún tipo.

- Configuración Pizarra Digital Interactiva (PDI): PDI (computadora, video-proyector, pizarra táctil) y acceso a Internet. Una 
configuración como ésta es la que Domingo y Marques (2011) llaman Aula 2.0.

- Configuración 3 Pizarras Digitales Interactivas (3PDI): Tres PDI (computadora, video-proyector, pizarra táctil) y acceso a Internet en todas.

De cada una de las configuraciones anteriores, hemos realizado un análisis de sus componentes y de las posibles situaciones didácticas en donde se pueden implementar (introducción al tema, desarrollo, ejercitación o práctica; aplicación o transferencia); grado de actividad de los estudiantes (alto, medio, bajo) y la actividad de los profesores (alto, medio, bajo). Esta información la presentamos en la tabla 1.

\section{Tabla 1. Componentes y situaciones didácticas en las cuales se pueden emplear}

\begin{tabular}{|l|l|c|c|}
\hline Configuración & $\begin{array}{c}\text { Situación didáctica } \\
\text { Introducción al tema, } \\
\text { desarrollo, ejercitación } \\
\text { aplicación }\end{array}$ & $\begin{array}{c}\text { Grado de actividad } \\
\text { de los estudiantes } \\
\text { (alto medio bajo) }\end{array}$ & $\begin{array}{c}\text { Grado de } \\
\text { participación del } \\
\text { profesor } \\
\text { (alto medio bajo) }\end{array}$ \\
\hline $\begin{array}{l}\text { Pizarra } \\
\text { Tradicional }\end{array}$ & - Introducción al tema & Bajo & Alto \\
\hline PD & $\begin{array}{l}\text { - Introducción al tema } \\
- \text { Aplicación }\end{array}$ & Bajo & Alto \\
\hline PD+PT & $\begin{array}{l}\text { - Introducción al tema } \\
\text { - Aplicación }\end{array}$ & Bajo & Alto \\
\hline L & $\begin{array}{l}\text { - Desarrollo } \\
- \text { Ejercitación } \\
\text { - Aplicación }\end{array}$ & Alto & Medio \\
\hline PDI & $\begin{array}{l}\text { - Introducción al tema } \\
- \text { Ejercitación } \\
- \text { Aplicación }\end{array}$ & Medio & Medio \\
\hline 3PDI & $\begin{array}{l}\text { - Introducción al tema } \\
- \text { Ejercitación } \\
- \text { Desarrollo } \\
- \text { Aplicación }\end{array}$ & Alto & Medio \\
\hline
\end{tabular}

La cantidad de posibles empleos de tecnología digital, estrategias didácticas y dinámicas entre el profesor y los estudiantes -además de las modalidades educativas (ver Ramírez-Martinell y Maldonado, 2014)es muy variada y las situaciones didácticas anteriores pueden servir para ilustrar la conveniencia del diseño didáctico y de las decisiones de 
inclusión de las TIC como uno de los distintos componentes de la configuración de la clase. No está de más mencionar que las configuraciones mencionadas no son únicas y que aunque las necesidades del tema sugieran el uso de ciertas tecnologías, la aplicación de otras distintas podrían bien justificarse.

Es importante comentar que ninguna de estas configuraciones es absolutamente superior a otra, pues su utilidad y conveniencia dependerá del uso que se le quiera dar en cada momento de una clase, marcado por determinada situación didáctica. Otra razón que puede favorecer la conveniencia de cada una, puede ser el nivel que se quiera tener en la actividad docente, los actores que participarán en ella, la cantidad de estudiantes y profesores y las condiciones de infraestructura tecnológica. Finalmente, la decisión crucial para escoger una configuración va a depender de la pregunta: ¿Qué buscamos en la clase? En dependencia de lo objetivo (presentación efectiva de contenido, participación activa de estudiantes que se involucren, etc.) debe escogerse la vía más adecuada.

\section{Uso de Pizarras Digitales Interactivas (PDI) en el contexto educativo}

Es sabido que la mayoría de las potencialidades de usar las PDI en el contexto educativo no son exclusivas de esta tecnología, ya que algunos de los beneficios se pueden atribuir a la didáctica de los profesores, al contenido que se emplean y al nivel de compromiso de los estudiantes. En esta sección, analizaremos algunos ejemplos concretos del empleo de las PDI y sus singularidades educativas con respecto a otras estrategias didácticas, recursos y configuraciones del aula.

Como se dijo en una sección anterior, es posible crear distintas configuraciones del aula, en función de la presencia o la ausencia de pizarra tradicional, video-proyector, computadora, bocinas, PDI, acceso - o no- a Internet, cámara de video, entre otros, o en función de las estrategias didácticas empleadas en la experiencia de aprendizaje y del grado de compromiso del profesor y de sus estudiantes.

La consideración de alguno de estos componentes o su combinación, propiciará el empleo de determinadas posibilidades didácticas. Para ejemplificar estas posibilidades, presentamos ocho situaciones en las que distintos elementos se articulan para poder así atender las necesidades de la mejor manera: 
1. Sensibilización de los estudiantes en el uso de redes sociales. Un profesor que quiere analizar con los estudiantes los peligros que podrían asociarse al empleo de redes sociales podría requerir de una computadora, un video-proyector y acceso a Internet, para poder explorar situaciones reales y pedirle a los estudiantes que compartan información de sus redes sociales. Para este caso, el uso de bocinas o cámaras de video parecieran ser poco necesarias.

2. Clase sobre una autopsia. Un profesor de medicina forense que quiera simular una autopsia podría requerir un programa controlable con los dedos que vaya mostrando los órganos internos del cuerpo mediante el uso de software de simulación, de un puntero o de una pantalla táctil y de un proyector. El acceso a Internet y el uso de un software de redes sociales parecieran no ser necesarios para esta situación didáctica.

3. Práctica de canto. Es probable que un profesor de canto, que quiera analizar con su grupo de estudiantes como influye la forma de los componentes de la boca (lengua, labios, etc.) en el sonido que se emite, necesite de una bocina y de una cámara de vídeo para filmar a sus estudiantes cantando. Para este caso, es probable que no se requiera del uso de Internet y de una pantalla táctil.

4. Clase de caligrafía en la pizarra tradicional. Un docente que quiere mejorar la caligrafía de nuevos docentes a la hora de escribir en una pizarra tradicional podría necesitar solamente la pizarra tradicional. Para este caso, el uso de la computadora y de sus demás sus accesorios pudiera ser redundante.

5. Clase de caligrafía en la pizarra táctil. Un docente que quiere analizar con los nuevos usuarios de las pizarras táctiles la influencia de la velocidad de escritura o de su postura al escribir podría diseñar una actividad empleando la pizarra táctil y una cámara de video para filmar el desempeño de los docentes.

6. Clase de mecanografía. Un entrenador de mecanografía que prepara a personas que deben digitalizar documentos manuscritos podría justificar el uso de un teclado en clase y no precisar de una pizarra tradicional ni de una pizarra táctil o de un mouse o acceso a Internet. No obstante, siempre se pueden pensar en otras posibilidades como utilizar un procesador de palabras, una cámara para filmar los desempeños y un proyector para mostrar el video. 
7. Situación "Bases de Datos". Un profesor quiere analizar diferentes formas de obtener una información de una base de datos, orientando a los estudiantes la elaboración de consultas para obtenerla y luego hacer un análisis comparativo de las soluciones en cuanto a eficacia y eficiencia. Para este caso, una computadora, un video-proyector y un PD serían deseables.

8. Situación "Ética". Un profesor quiere analizar diferentes aspectos éticos que pueden estar afectando una decisión que tiene que tomar una empresa considerando varias dimensiones como las influencia en los trabajadores, en la competencia, en los consumidores y en la comunidad donde está ubicada la empresa. Quiere dividir el aula en grupos que analicen cada aspecto y luego integrar y discutir los resultados, es posible que esta situación se pudiera resolver en un laboratorio.

A partir de las situaciones anteriores y de las distintas configuraciones en que se enfoca el análisis, se presenta seguidamente una valoración de hasta qué punto cada configuración es conveniente o no para cada situación. Para organizar de manera compacta esta valoración, hemos organizado la información en una matriz de doble entrada, con las ocho situaciones didácticas previamente descritas; además, de la valoración de la configuración (I - Incumplible, P - Parcialmente cumplible, C-Cumplible o E- Excesivo) a partir de su viabilidad. Por ejemplo, para el caso de la clase acerca redes sociales, consideramos que el uso de una PT permitiría alcanzar el objetivo didáctico de manera parcial mientras que el uso de una PD, de una PD+PT o de una PDI sería lo más adecuado, ya que un L o 3PDI podrían resultar excesivos.

- Incumplible (I). El objetivo esencial no se puede cumplir, porque los medios no lo permiten.

- $\quad$ Cumplible parcialmente (P). Aunque no se puede cumplir cabalmente el objetivo, hay partes de él que son logrables o hasta cierto grado.

- $\quad$ Cumplible (C). Puede cumplirse el objetivo esencial de la situación.

- Excesivo (E). Puede irse más allá del objetivo de la situación, logrando nuevos efectos positivos en el proceso de enseñanza-aprendizaje. 
Tabla 2. Situaciones y configuraciones

\begin{tabular}{|l|c|c|c|c|c|c|}
\hline \multirow{2}{*}{ Situación } & \multicolumn{7}{|c|}{ Configuraciones } \\
\cline { 2 - 7 } & PT & PD & PD+PT & L & PDI & 3PDI \\
\hline Redes sociales & P & C & C & E & C & E \\
\hline Autopsia & $\mathrm{I}$ & $\mathrm{P}$ & $\mathrm{P}$ & $\mathrm{E}$ & $\mathrm{E}$ & $\mathrm{E}$ \\
\hline Canto & $\mathrm{P}$ & $\mathrm{C}$ & $\mathrm{C}$ & $\mathrm{E}$ & $\mathrm{C}$ & $\mathrm{E}$ \\
\hline Caligrafía 1 & $\mathrm{C}$ & $\mathrm{I}$ & $\mathrm{E}$ & $\mathrm{I}$ & $\mathrm{E}$ & $\mathrm{E}$ \\
\hline Caligrafía 2 & $\mathrm{P}$ & $\mathrm{I}$ & $\mathrm{C}$ & $\mathrm{I}$ & $\mathrm{E}$ & $\mathrm{E}$ \\
\hline Mecanografía & $\mathrm{I}$ & $\mathrm{P}$ & $\mathrm{P}$ & $\mathrm{C}$ & $\mathrm{P}$ & $\mathrm{C}$ \\
\hline Bases de Datos & $\mathrm{P}$ & $\mathrm{C}$ & $\mathrm{C}$ & $\mathrm{E}$ & $\mathrm{C}$ & $\mathrm{E}$ \\
\hline Ética & $\mathrm{C}$ & $\mathrm{C}$ & $\mathrm{C}$ & $\mathrm{E}$ & $\mathrm{C}$ & $\mathrm{E}$ \\
\hline
\end{tabular}

De la tabla dos, podemos ver que hay situaciones que se benefician notablemente con la presencia de PDI (Configuraciones PDI y 3PDI) como son las situaciones "autopsia" y las dos de c" debido a que la PDI permite combinar las ventajas de contar con las capacidades de una computadora y con una interfaz más libre (que rebase lo que el teclado y el mouse proporcionan). En el caso de "Autopsia", podría permitir tener una experiencia cercana a la real que podría tener un médico forense ante un cadáver, mientras que en "Caligrafía" permitiría analizar la forma particular de escritura de cada nuevo docente y su influencia en la calidad caligráfica.

Sin embargo, hay situaciones en que la PDI no aportaría tanto respecto a la configuración $\mathrm{PD}$, como puede ser el mencionado caso de "Redes sociales" o las situaciones didácticas de "Canto", "Bases de Datos" y "Ética". En el caso de "Canto", lo esencial parece estar en la posibilidad de la computadora para filmar la forma de cantar de los estudiantes para analizarla luego. Aquí parece ser más interesante la presencia de varias computadoras (configuraciones L y 3PDI) que permitirían filmar y analizar en paralelo a varios estudiantes. Algo parecido sucede con el caso "Bases de Datos" donde parece más interesante la posibilidad de contar con varias computadoras en las que en paralelo (individual o grupalmente) se vayan probando las versiones elaboradas por los estudiantes, o en el de "Ética" para ir avanzando en paralelo en las distintas dimensiones de análisis. En el caso "Redes sociales", lo interesante no estaría tanto en el acceso manual para navegar en Internet (que solo produciría un efecto Hawthorne) sino en la posibilidad de 
navegación en paralelo de manera independiente de cada grupo, para luego compartir la experiencia.

En el caso de "Base de Datos", la presencia del proyector (configuraciones PD, PD+PT, PDI y 3PDI) puede ser interesante para hacer un análisis global y comparativo de las respuestas. Lo mismo pasa en el caso de "Ética" y para integrar y analizar entre todos lo trabajado en cada grupo o individualmente o en el caso "Redes Sociales" para compartir lo visto.

Sobre el análisis anterior podrían hacerse objeciones en dos direcciones, tanto en el sentido de usar menos o usar más la tecnología. En el sentido de usar menos tecnología, para el caso de "Ética", podría argumentarse que usando lápiz y pizarra tradicional podría lograrse el mismo resultado de trabajo colaborativo y posterior integración. Igualmente, la situación "Redes Sociales" podría enfocarse analizando material impreso traído por el profesor. Sin embargo, en este último caso, el análisis estaría restringido. Además, en ambos llevaría más tiempo el proceso de socialización de lo trabajado en cada grupo (habría que copiarlo en la pizarra), sería más difícil la reelaboración de las ideas (usando borrador y marcador) que lo que podría lograrse con la ayuda de la computadora en este sentido, así como la limitación para usar material multimedia como soporte de las ideas.

En el sentido contrario (hacia usar más tecnología), podrían hacerse objeciones en todos los casos, mostrando otras posibilidades que puede aportar la presencia de más componentes. Por ejemplo, podría objetarse que si se contara con programas avanzados que puedan mostrar la estructura de cuerdas vocales navegando por ellas con control manual de una PDI, entonces la situación "Canto" podría llevarse a una dimensión más avanzada. Sin embargo, esto va más allá de lo planteado en este caso y lo acercaría a la situación "Autopsia".

La situación "Mecanografía" es una situación donde lo que parece más relevante es la presencia de varias computadoras (más concretamente de los teclados) en que son ventajosas las configuraciones L y 3PDI (en menor medida). Además de estas configuraciones, la presencia de al menos una computadora (configuraciones PD, PD+PT, y PDI) podría ser una solución aproximada porque podrían ir pasando por la computadora cada uno de los estudiantes.

El análisis anterior está lejos de ser exhaustivo, pero ilustra situaciones donde cada uno de los componentes y la sinergia entre ellos 
parece ser más o menos relevante. A continuación, ponemos otros cuatro ejemplos de situaciones que pudieran verse como análogas a las primeras ocho descritas.

1. Un profesor de Mecánica que quiere analizar con sus estudiantes las partes de un motor o un profesor de computación que pretende estudiar la arquitectura y organización de la computadora podría estar en presencia de una situación parecida a la situación "Autopsia". Estos son casos en que el aspecto táctil de la pizarra (o la superficie de proyección que sea) puede cobrar relevancia si se cuenta con los programas adecuados para emplearla, dada la presencia de un soporte físico de la materia sobre la que se está haciendo el estudio y que podría convenir el efecto del tacto como órgano sensorial. Otros casos parecidos podrían darse el contexto de geología, construcciones, etc.

2. Podría creerse que estaría en el mismo caso un profesor de Historia que quiere usar un mapa como interfaz sobre las que irá apareciendo distintos elementos de históricos, al estilo de lo que se proporciona con las herramientas de "tinta mágica" y "caja mágica”. Sin embargo, no parece tan esencial aquí el aspecto táctil, sino más bien, el carácter interactivo que aporta la computadora. Esta situación estaría más cercana a la situación "Redes Sociales" donde la interacción es lo más importante y los recursos que se van descubriendo más allá del efecto Hawthorne o de novedad que puede producir lo táctil.

3. Un profesor de literatura que pretende estudiar una obra literaria o un profesor de mercadotecnia que quiere diseñar una campaña de mercado podría estar en una situación similar a la situación "Ética" o la situación "Bases de Datos", donde convendría dividir el aula para enfocar el análisis en aspectos diferentes, usar material multimedia para elaboración independiente, buscar información en Internet y luego hacer un análisis colectivo. En este caso, que parece ser un trabajo más conceptual puede que lo táctil sea menos relevante. Claro está, si el diseño puede ayudarse con programas que soporten interfaces táctiles, por ejemplo, para el diseño de formas y texturas el carácter táctil podría ser enriquecedor.

4. Diversos casos de enseñanza artística, de idiomas y de enseñanza especial podría caer en situaciones similares a las situaciones 
"Canto", las dos "Caligrafía" y "Mecanografía" según sea de lo que se trate (un instrumento musical objeto de estudio, la buena pronunciación de palabras, la escritura Braille, etc.).

Es imposible hablar de las distintas configuraciones y su viabilidad, pero lo que sí podemos hacer es enfatizar que la existencia de un medio, no está relacionada directamente con su empleo concreto. Si un profesor (ya sea para una actividad concreta, o de manera general) quiere emplear un enfoque centrado en la enseñanza más que en el aprendizaje, si quiere tener un control total de lo que ocurre en el aula, y su objetivo central es tener la atención de todos los estudiantes enfocada en él mismo como fuente de conocimiento para los estudiantes, podría ser que los medios disponibles sean ignorados o incluso que sean un freno para sus metas. Por ejemplo, una configuración L o 3PDI sería muy compleja de manejar, ya que cada estudiante tendería a usar libremente su computadora, caminando en sentidos diferentes a lo que el profesor pretende. Al respecto, autores como Jara, Santa, Zamora, Villalba, Guirao y Pinazo (2011) y Glover, Miller, Averis y Door (2007) han dejado claro que es muy importante usar al máximo las capacidades de las tecnologías digitales disponibles con fines educativos, argumentando que es común encontrar a profesores que siguen basando su clase en presentaciones con diapositivas sin emplear PDI. Debe quedar claro que muchas veces esto está limitado por los medios y programas con los que se cuenta. Sin embargo, también una causa puede ser el tiempo mayor que implica la preparación de una clase que emplee a fondo todas las posibilidades y diferencias en aprendizaje de cada uno según su singularidad. Esta razón fue planteada por Gallego, Cacheiro y Dulac (2009) y por Noda (2009); y como lo hemos dicho la configuración deberá ir acompañada de la reflexión sobre el análisis de sus componentes $\mathrm{y}$ de las posibles situaciones didácticas en donde se pueden implementar las PDI (como en la introducción al tema, su desarrollo, ejercitación o práctica; en la aplicación o transferencia); además de la consideración del grado de actividad (alto, medio, bajo) tanto de los estudiantes como de los profesores y la actividad de los profesores (alto, medio, bajo).

Otro aspecto a considerar es que no se puede ver siempre como una ganancia el tener varias computadoras o PDI (configuraciones L y 3PDI), pues algunos autores han observado que aunque puede ser ventajosa la posibilidad de tener varias PDI o de dividir la pantalla de 
esta en varias partes para ver el trabajo de varios de los actores universitarios; Jara, Santa, Zamora, Villalba, Guirao y Pinazo (2011) han observado que en algunos casos, más de dos estudiantes y el profesor cuando actúan simultáneamente en la PDI pueden provocar problemas de atención y concentración.

En general, consideramos necesario estudiar los componentes, las situaciones didácticas en donde se vayan a emplear, los grados de actividad de los profesores y estudiantes; las configuraciones y las singularidades de las PDI con respecto a sus impactos y cambios en el proceso de enseñanza aprendizaje. De hecho, algunos autores (Glover, Miller, Averis y Door, 2005; 2007) han observado que hay distintos aspectos que se mencionan en los materiales ofertados por los proveedores de la infraestructura tecnológica que aún no han recibido atención en las investigaciones. Solo para aludir a un ejemplo, algunos productos PDI mencionan que disponen de la posibilidad de imprimir lo trabajado en el aula; sin embargo, no está claro lo que se gana de esto, desde el punto de vista pedagógico. Otro aspecto que ha sido poco estudiado es la diferencia de percepción sobre las PDI a diferentes edades. Consideramos que el método y las valoraciones presentadas en este trabajo avanzan un paso en la dirección de ese necesario estudio.

\section{Conclusiones}

En este trabajo hemos revisado algunos de los trabajos publicados sobre el empleo de las PDI; adicionalmente, hemos presentado un grupo de situaciones que se pueden presentar en un aula para un docente que tiene determinadas intenciones educativas. En estas situaciones, en las que hemos tratado de buscar un espectro amplio de temas, asimismo hemos podido mostrar cuáles de los componentes de las Pizarras Digitales Interactivas juegan un rol de mayor importancia para el logro de determinados objetivos de aprendizaje y cuáles no. En algunos casos, el carácter táctil de la pizarra ha demostrado ser más relevante que en otros, aunque de forma general la creatividad de los docentes puede servir para vislumbrar oportunidades educativas que empleen todas las potencialidades de que se disponga.

Las PDI pueden aumentar la motivación de docentes y estudiantes, pero esto pasa en primera instancia por un cambio en los estilos de enseñanza y de la necesidad de incorporar nuevas técnicas que permitan 
al docente enfocarse en la práctica y la participación activa durante las sesiones de trabajo en el aula. Aunque puede haber resistencia al uso de PD y PDI por la necesidad implícita de aprender sobre el uso del dispositivo, consideramos que el foco de atención debería estar en la planeación y en el análisis de las situaciones didácticas, ya que las cuestiones técnicas se pueden resolver con apoyo de personal o inclusive de los mismos estudiantes. El reto mayor para lograr que las PDI produzcan un mejoramiento real en la experiencia educativa va más allá de la adquisición y el conocimiento de las tecnologías. Para lograr un uso efectivo de las PDI, hay que tener en cuenta aspectos tales como la configuración de medios al alcance del docente, el grado de actividad que se pretende lograr con los estudiantes, además de las situaciones y momentos en los que se quiere emplear el nuevo dispositivo.

A partir de los casos analizados, puede notarse que vale la pena utilizar PDI en diversas situaciones porque realmente pueden producir una mejoría en la experiencia educativa. Sin embargo, no quiere decir que sean la solución a todas las situaciones pedagógicas. De hecho, hay casos entre los presentados en que las PDI aportaron poco o nada a la concreción de los objetivos docentes, lo cual realza la necesidad de un mayor estudio de las condiciones en que las PDI son realmente útiles, en consecuencia, se debe fomentar su uso cuando provoquen un aporte real.

Con esta reflexión pretendemos avanzar un paso en la dirección del entendimiento y comprensión de las situaciones en que conviene o no usar las PDI; a la vez, pretendemos proponer líneas de procuración e investigación como pueden ser la elaboración de recomendaciones metodológicas para el uso correcto de las PDI, la forma en que se pueden combinar con otros medios didácticos y el uso que los estudiantes dan a las PDI, entre otras.

\section{Referencias bibliográficas}

Armstrong, V., Barnes, S., Sutherland, R., Curran, S., Mills, S. y Thompson, I. (2005). Collaborative research methodology for investigating teaching and learning: the use of interactive whiteboard, technology. Educational Review, 57 (4), 457-469.

Domingo, M. y Marques, P. (2011). Aulas 2.0 y uso de las TIC en la práctica docente. Comunicar, XIX (37), 169-174. 
Gallego, G., Cacheiro, M. L. y Dulac, J. (2009). La pizarra digital interactiva como recurso docente. Revista Electrónica Teoría de la Educación: Educación y Cultura en la Sociedad de la Información, 10 (2), 127-145.

Glover, D., Miller, D., Averis, D. y Door, V. (2005). The Interactive Whiteboard: a literature survey. Technology, Pedagogy and Education, 14 (2), 155-170.

Glover, D., Miller, D., Averis, D. y Door, V. (2007). The evolution of an effective pedagogy for teachers using the interactive whiteboard in mathematics and modern languages: an empirical analysis from the secondary sector. Learning, Media and Technology, 32 (1), 5-20.

Guerrero, J. (2009). Introducción de las pizarras digitales interactivas en la enseñanza presencial y no presencial, IV Jornadas Nacionales sobre el Espacio Europeo de Educación Superior, Murcia, España.

Hervás, C., Toledo, P. y González, M.C. (2010). La utilización conjunta de la pizarra digital interactiva y el sistema de participación Senteo: una experiencia universitaria. Pixel-Bit. Revista de Medios y Educación, Enero (36), 203-214.

Jara, A.J., Santa, J., Zamora, M. A., Villalba, G., Guirao, A. y Pinazo, R. (2011). Transferring experience in digital boarda and classroom response system form the Computer Science Faculty to High Schools. Proceedings of INTED2011 Conference, Valencia, Spain.

Marques, P. (2006), Pizarra Digital (1a Ed.). Barcelona: Edebé.

Marques, P., Casals, P. (2002). La pizarra digital en el aula de clase, una de las tres bases tecnológicas de la escuela del futuro. Revista Fuentes, 4 (1), 53-62.

Noda, A. (2009). Pizarra digital interactiva en aulas de matemáticas. Números - Revista didáctica de las matemáticas, 72, 121-127.

Passey, D. (2009). Evolving Forms of Visualisation for Presenting and Viewing Data. En C. O'Mahony, A. Finegan, A. J. Visscher y A. Tatnall (Eds.), Evolution of Information Technology in Educational Management, (pp. 155-167). IFIP International Federation for Information Processing, Boston: Springer.

Quiroz, M. (2012). Entrenamiento Básico, Manual de Guía para el docente, Utilización del Software Educativo ActivInspire (ActivInspire Studio - ActivInspire Primary). Latin America \& Caribbean. Promethean Inc. 
Ramírez-Martinell, A. y Maldonado, A. G. (2015) Multimodalidad en educación superior. En A. Ramírez-Martinell y M.A. Casillas (coord.). Háblame de TIC, volumen 2: Internet en Educación Superior. Córdoba Argentina: Editorial Brujas. 